\title{
Glucose Metabolic Profile by Visual Assessment Combined with Statistical Parametric Mapping Analysis in Pediatric Patients with Epilepsy
}

\author{
Yuankai Zhu*1-4, Jianhua Feng ${ }^{* 5}$, Shuang Wu*1-4, Haifeng Hou*1-4, Jianfeng Ji ${ }^{1-4}$, Kai Zhang ${ }^{1-4}$, Qing Chen ${ }^{1-4}$, \\ Lin Chen ${ }^{1-4}$, Haiying Cheng ${ }^{5}$, Liuyan $\mathrm{Gao}^{5}$, Zexin Chen ${ }^{6}$, Hong Zhang ${ }^{1-4}$, and Mei Tian ${ }^{1-4}$ \\ ${ }^{1}$ Department of Nuclear Medicine, The Second Hospital of Zhejiang University School of Medicine, Hangzhou, China; ${ }^{2}$ Zhejiang \\ University Medical PET Center, Hangzhou, China; ${ }^{3}$ Institute of Nuclear Medicine and Molecular Imaging of Zhejiang University, \\ Hangzhou, China; ${ }^{4}$ Key Laboratory of Medical Molecular Imaging of Zhejiang Province, Hangzhou, China; ${ }^{5}$ Department of \\ Pediatrics, The Second Hospital of Zhejiang University School of Medicine, Hangzhou, China; and ${ }^{6}$ Department of Clinical \\ Epidemiology \& Biostatistics, The Second Hospital of Zhejiang University School of Medicine, Hangzhou, China
}

PET with ${ }^{18} \mathrm{~F}-\mathrm{FDG}$ has been used for presurgical localization of epileptogenic foci; however, in nonsurgical patients, the correlation between cerebral glucose metabolism and clinical severity has not been fully understood. The aim of this study was to evaluate the glucose metabolic profile using ${ }^{18} \mathrm{~F}-\mathrm{FDG}$ PET/CT imaging in patients with epilepsy. Methods: One hundred pediatric epilepsy patients who underwent ${ }^{18} \mathrm{~F}-\mathrm{FDG}$ PET/CT, MRI, and electroencephalography examinations were included. Fifteen age-matched controls were also included. ${ }^{18} \mathrm{~F}-\mathrm{FDG}$ PET images were analyzed by visual assessment combined with statistical parametric mapping (SPM) analysis. The absolute asymmetry index (|AI|) was calculated in patients with regional abnormal glucose metabolism. Results: Visual assessment combined with SPM analysis of ${ }^{18} \mathrm{~F}$ FDG PET images detected more patients with abnormal glucose metabolism than visual assessment only. The $\mid$ Al $\mid$ significantly positively correlated with seizure frequency $(P<0.01)$ but negatively correlated with the time since last seizure $(P<0.01)$ in patients with abnormal glucose metabolism. The only significant contributing variable to the $|\mathrm{Al}|$ was the time since last seizure, in patients both with hypometabolism $(P=0.001)$ and with hypermetabolism $(P=0.005)$. For patients with either hypometabolism $(P<0.01)$ or hypermetabolism $(P=0.209)$, higher $|\mathrm{Al}|$ values were found in those with drug resistance than with seizure remission. In the post-1-y follow-up PET studies, a significant change of |AI| (\%) was found in patients with clinical improvement compared with those with persistence or progression $(P<0.01)$. Conclusion: ${ }^{18} \mathrm{~F}-\mathrm{FDG}$ PET imaging with visual assessment combined with SPM analysis could provide cerebral glucose metabolic profiles in nonsurgical epilepsy patients. |Al| might be used for evaluation of clinical severity and progress in these patients. Patients with a prolonged period of seizure freedom may have more subtle (or no) metabolic abnormalities on PET. The clinical value of PET might be enhanced by timing the scan closer to clinical seizures.

Received Nov. 18, 2016; revision accepted Dec. 15, 2016.

For correspondence or reprints contact: Mei Tian, Department of Nuclear Medicine, The Second Hospital of Zhejiang University, 88 Jiefang Rd., Hangzhou, Zhejiang 310009, China. E-mail: meitian@zju.edu.cn

*Contributed equally to this work

Published online Jan. 19, 2017.

COPYRIGHT (c) 2017 by the Society of Nuclear Medicine and Molecular Imaging.
Key Words: epilepsy; glucose metabolism; positron emission tomography (PET); statistical parametric mapping (SPM)

J Nucl Med 2017; 58:1293-1299

DOI: 10.2967/jnumed.116.187492

$\mathbf{E}$ pilepsy is considered one of the most common serious neurologic conditions, affecting 65 million people of all ages worldwide (1). Most patients with epilepsy fall into the pediatric group, and approximately $20 \%$ of these patients have medically intractable epilepsy (2). So far, electroencephalogram (EEG) evaluation remains the most widely used method in clinical practice to classify the type of seizure in patients with epilepsy; however, the frequency of epileptiform EEG discharges is weakly related to severity of epilepsy (3). For the localization of seizure onset and the identification of the pathologic findings underlying the epileptic brain tissue in drug-resistant epilepsy patients, a structural neuroimaging technology, MRI, has obvious clinical advantages. MRI is effective for identifying intracranial abnormalities commonly associated with chronic focal epilepsy (4). However, not all MRI abnormalities, including hippocampal sclerosis, cavernomas, gliomas, and malformations, cause seizures and not all seizures originate from identified structural cerebral abnormalities. MRI fails to reveal epileptic foci in 20\%-30\% of temporal lobe epilepsy (TLE) and $20 \%-$ $40 \%$ of extra-TLE patients, despite advancement of functional MRI and MR spectroscopy $(5,6)$.

Recently, interictal PET with ${ }^{18} \mathrm{~F}-\mathrm{FDG}$ has been used as a powerful neuroimaging technology for presurgical evaluation of epileptogenic foci (7-9) and has demonstrated a sensitivity of 70\%$90 \%$ in TLE and 33\%-67\% in extra-TLE patients, depending on the localization of the focus (10-12). The highest clinical benefit of ${ }^{18}$ F-FDG PET can be achieved in patients with MRI-negative TLE, for whom ${ }^{18} \mathrm{~F}$-FDG PET correctly lateralized the lesion in $80 \%$ of the cases (13). However, for the nonsurgical patients, especially for the nonsurgical pediatric children, the clinical diagnostic and prognostic values of ${ }^{18} \mathrm{~F}$-FDG PET remain unclear.

Therefore, we hypothesized that visual assessment combined with statistical parametric mapping (SPM) analysis (or SPM-based visual analysis) of ${ }^{18}$ F-FDG PET images could be useful for evaluating the correlation between cerebral metabolism and clinical severity or progress in epilepsy patients. The aim of this study was 
to evaluate the glucose metabolic profile using ${ }^{18} \mathrm{~F}-\mathrm{FDG}$ PET/CT imaging in patients with nonsurgical epilepsy.

\section{MATERIALS AND METHODS}

\section{Subjects}

We retrospectively reviewed 142 pediatric epilepsy patients who had undergone ${ }^{18} \mathrm{~F}$-FDG PET, EEG, and MRI examinations to detect epileptogenic areas. The inclusion criteria were as follows: clinical diagnosis of epilepsy (14); age between 7 and $14 \mathrm{y}$; detailed information of EEG and MRI; the last seizure occurring more than $24 \mathrm{~h}$ before ${ }^{18} \mathrm{~F}-\mathrm{FDG}$ PET study (15); and completion of 12-mo clinical follow-up after ${ }^{18} \mathrm{~F}-\mathrm{FDG}$ PET imaging. Patients were excluded if they had no data from one of the modalities (EEG, MRI, or PET). Other exclusion criteria consisted of medical illness with central nervous system impact other than epilepsy, for example, head trauma, encephalitis, tumor, infarct, or porencephaly, because these obvious lesions preclude the normalization of ${ }^{18} \mathrm{~F}-\mathrm{FDG}$ PET images into standard template in SPM analysis. According to the type and number of seizures experienced in the $12 \mathrm{mo}$, epilepsy patients were divided into the severe, moderate, or mild groups (16). Patients who had more than 20 seizures per year were classified as "severe," and those without seizures in the past 12 mo were considered as "mild." At the end of the clinical follow-up, patients were considered to be in remission if they had no seizures during the past 12 mo or considered drug-resistant if they failed to achieve sustained seizure freedom despite adequate antiepileptic drugs (AEDs) $(17,18)$. One hundred epilepsy patients (46 girls and 54 boys; mean age $\pm \mathrm{SD}, 10.4 \pm 2.0$ y) were included in this study. At the time of the PET scan, 49 patients received 1 type of AED, 29 patients received 2 AEDs, and 8 patients required treatment with 3 or more AEDs. The most frequently used AED was oxcarbazepine $(n=55)$, followed by levetiracetam $(n=22)$, topiramate $(n=21)$, valproate $(n=19)$, lamotrigine $(n=10)$, clonazepam $(n=4)$, and carbamazepine $(n=1)$. All patients underwent at least 1 prolonged ( $24 \mathrm{~h}$ ) EEG monitoring to confirm the localization of the epileptic foci. Long-term video EEG was performed in the evaluation of 23 patients who presented difficulties in localization after clinical assessment, prolonged EEG, and MRI. Fifteen nonepilepsy patients ( 9 girls and 6 boys; mean age, $11.1 \pm 3.1$ y) who had no history of neurologic disorders, psychiatric illnesses, chemotherapy, or radiotherapy were included as the age-matched control subjects for SPM analysis. This age-matched control group consisted of 6 patients with extracranial lymphoma and 9 with bone neoplasm. The institutional review board approved this retrospective study, and the requirement to obtain informed consent was waived (ChiCTR-ORC-16009167).

\section{PET Image Analysis}

PET/CT brain images were acquired on a PET/CT scanner (Biograph $\mathrm{mCT}$; Siemens Medical Solutions) at $40 \mathrm{~min}$ after intravenous injection of ${ }^{18}$ F-FDG $(3.7 \mathrm{MBq} / \mathrm{kg})(19,20)$. PET and MR images were coregistered using the vender-provided Fusion Registration Software implemented in the syngo MultiModality Workplace (Siemens Medical Solutions).

Visual Assessment. ${ }^{18} \mathrm{~F}-\mathrm{FDG}$ PET images were visually evaluated by 2 nuclear medicine specialists who were unaware of the findings of EEG and MRI. The glucose metabolic patterns (hypometabolism, hypermetabolism, or normal metabolism) of 16 major brain regions (8 in each hemisphere, corresponding to lateral and medial surfaces of the 4 lobes [frontal, temporal, parietal, occipital]) were evaluated and reported separately. Discordant results were reviewed by both experts to reach a consensus.

SPM Analysis. Static ${ }^{18}$ F-FDG PET image volumes were analyzed using the SPM8 software package developed at the Wellcome Department of Cognitive Neurology, Institute of Neurology (21). ${ }^{18}$ F-FDG PET image volumes were spatially normalized into standard stereotactic space using an in-house pediatric PET template. A gaussian kernel of $16 \mathrm{~mm}$ in full width at half maximum was used to smooth the data for statistical analysis. Individual SPM analysis was performed using an age-matched control group. Increased or decreased metabolism was regarded as statistically significant if the uncorrected $P$ value was under 0.001 , with a cluster level above 100 voxels.

Visual Assessment Combined with SPM Analysis. Visual assessment combined with SPM analysis was performed for reanalysis of all the PET images. A further semiquantitative analysis, absolute asymmetry index $(|\mathrm{AI}|)$, was performed to evaluate the intensity of the metabolic abnormality in brain regions established using this combined assessment. In patients with more than 1 cortical area showing hypo- or hypermetabolism, the region with the most severe abnormality was selected for the calculation of $|\mathrm{AI}|$. According to a previous study (22), data were collected as $\mathrm{nCi} / \mathrm{cm}^{3}$ tissue for each region of interest with a $48 \mathrm{~mm}^{2}$ circle in the original PET scans, and $|\mathrm{AI}|$ was calculated using the following formula: $|\mathrm{AI}|=\mid($ left - right $) /([$ left + right $] / 2) \mid$.

\section{Statistical Analysis}

Values are reported as mean \pm SD. All data were analyzed by the SPSS software (version 20.0; IBM SPSS Statistics). Comparison of sex was performed using the $\chi^{2}$ test and concordance between visual assessment and SPM analysis using the $\kappa$ test. The 2-sample $t$ test was used to compare age and age of onset. Comparisons of seizure severity, seizure frequency, duration of epilepsy, number of AEDs, and time since last seizure were performed using the Mann-Whitney test. Comparisons of $|\mathrm{AI}|$ among groups were performed using either the Mann-Whitney test or the Kruskal-Wallis test, and followed by a post hoc test when appropriate. Analysis of correlation was performed using Spearman correlation coefficients. Stepwise multivariate linear regression analysis was conducted to determine the independent contribution of individual variables (age, age of onset, duration of epilepsy, seizure frequency, time since last seizure, and number of AEDs) to the $|\mathrm{AI}|$. A $P$ value of less than $0.05(P<0.05)$ was considered statistically significant.

\section{RESULTS}

After careful retrospective reviewing of EEG, MRI, and PET results and clinical follow-up, a total of 100 patients were included in this study. Epileptiform activity was noted on the EEG of 86 patients $(86 \%)$, with $76(76 \%)$ showing focal epileptiform abnormalities. Slowing was noted on the EEG in only 5 patients, 2 of whom were focal and 3 generalized. The EEG and ${ }^{18} \mathrm{~F}-\mathrm{FDG}$ PET results were both abnormal in 75 patients $(75 \%)$ and both normal in $5(5 \%)$ (Table 1). The EEG results were significantly associated with PET findings $(\kappa=0.237, P=0.008)$. In contrast, the EEG and MRI results were both abnormal in 37 patients $(37 \%)$ and both normal in 7 (7\%). The association between EEG and MRI findings was not significant ( $\kappa=0.051, P=0.279$ ).

\section{TABLE 1}

Comparisons of EEG, ${ }^{18} \mathrm{~F}-\mathrm{FDG}$ PET, and MRI Findings in Pediatric Epilepsy Patients $(n=100)$

\begin{tabular}{|c|c|c|c|c|c|}
\hline \multirow[b]{2}{*}{ EEG findings } & \multicolumn{2}{|c|}{$\begin{array}{l}{ }^{18} \mathrm{~F}-\mathrm{FDG} \text { PET } \\
\text { findings }\end{array}$} & \multicolumn{2}{|c|}{$\mathrm{MRI}^{\text {findings }}{ }^{\dagger}$} & \multirow[b]{2}{*}{ Total } \\
\hline & Normal & Abnormal & Normal & Abnormal & \\
\hline Normal & 5 & 4 & 7 & 2 & 9 \\
\hline Abnormal & 16 & 75 & 54 & 37 & 91 \\
\hline Total & 21 & 79 & 61 & 39 & 100 \\
\hline \multicolumn{5}{|c|}{$\begin{array}{l}{ }^{{ }^{*} \mathrm{~K}}=0.237, P=0.008 . \\
{ }^{{ }} \mathrm{K}=0.051, P=0.279 .\end{array}$} & \\
\hline
\end{tabular}


TABLE 2

Comparison between Visual Assessment and SPM Analysis of ${ }^{18}$ F-FDG PET Studies in Pediatric Epilepsy Patients $(n=100)$

\begin{tabular}{|c|c|c|c|c|}
\hline \multirow[b]{2}{*}{ Visual assessment } & \multicolumn{3}{|c|}{ SPM analysis } & \multirow[b]{2}{*}{ Total } \\
\hline & Normal & Hypometabolism & Hypermetabolism & \\
\hline Normal & 21 & 6 & 3 & 30 \\
\hline Hypometabolism & 8 & 41 & 14 & 63 \\
\hline Hypermetabolism & 0 & 0 & 7 & 7 \\
\hline Total & 29 & 47 & 24 & 100 \\
\hline $\mathrm{K}=0.483, P<0.001$. & & & & \\
\hline
\end{tabular}

\section{Visual Assessment and SPM Analysis of ${ }^{18}$ F-FDG PET Images}

Abnormal cerebral metabolism was observed by visual assessment in 70 patients $(70 \%)$, including 63 with hypometabolism and 7 with hypermetabolism (Table 2). SPM analysis identified abnormal metabolism in 71 patients ( $71 \%$ ), including 47 with hypometabolism and 24 with hypermetabolism. Among the 24 hypermetabolic patients, hypermetabolism associated with hypometabolism was detected in 14 patients using SPM analysis; however, primary visual assessment demonstrated only hypometabolic regions in these patients.

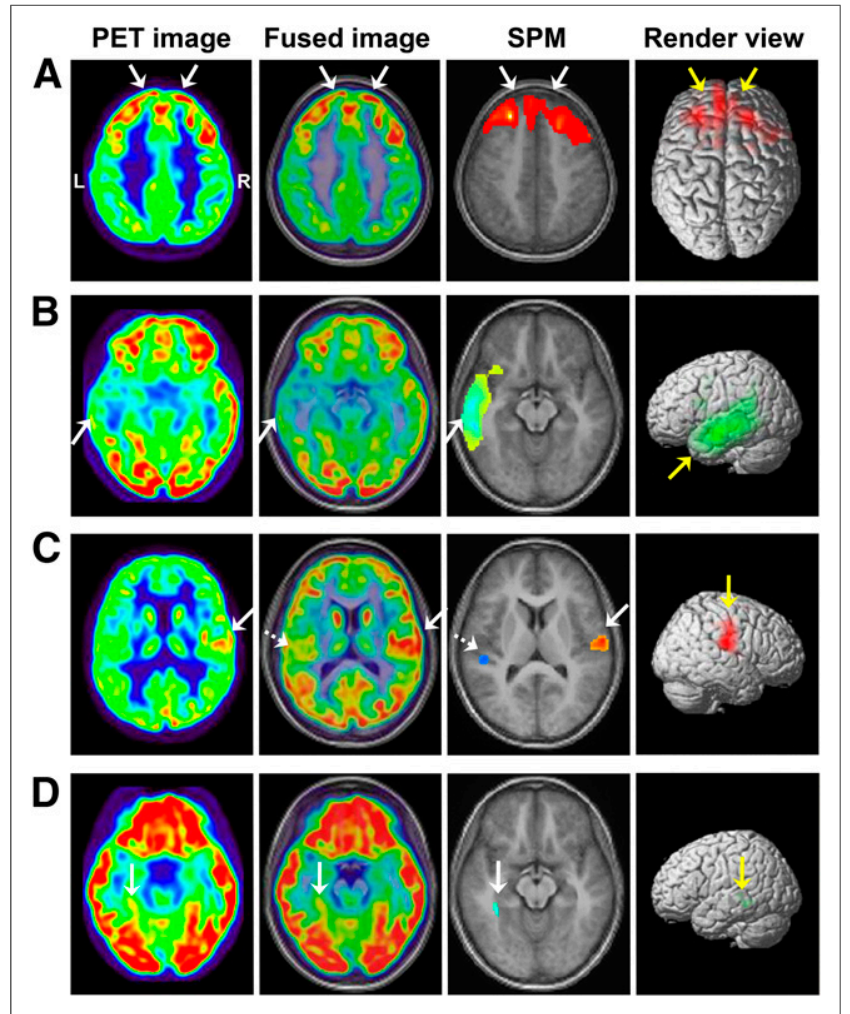

FIGURE 1. Comparison between visual and SPM analysis of ${ }^{18} \mathrm{~F}-\mathrm{FDG}$ PET images. (A) Hypermetabolism in bilateral frontal lobes (solid arrow) was detected by both visual and SPM analysis. (B) Hypometabolism in left temporal lobe (solid arrow) was detected by both visual and SPM analysis. (C) Hypometabolism was found in left rolandic area (dashed arrow) by visual assessment, and hypermetabolic region was further identified in right rolandic area (solid arrow) by SPM analysis. (D) Hypometabolic region undetected by visual assessment was identified in left mesial temporal lobe (solid arrow) by SPM analysis.
A concordant result between the visual and SPM analyses was obtained in 69 patients $(\kappa=0.483, P<0.001)$. Representative images were shown in Figure 1.

\section{Correlation Between Clinical Characteristics and Glucose Metabolism}

The analysis of correlation between clinical characteristics and glucose metabolism was based on the combined visual and SPM analysis. Patients with abnormal glucose metabolism had a significantly shorter time period since last seizure $(P<0.05)$ and a greater severity of epilepsy $(P<0.05)$ and seizure frequency $(P<0.05)$ than those with normal metabolism (Table 3). No significant differences were found in sex, age, age of onset, duration of disease, or

\section{TABLE 3}

Clinical Characteristics and ${ }^{18}$ F-FDG PET Findings in Pediatric Epilepsy Patients $(n=100)$

\begin{tabular}{llcc}
\hline \multicolumn{1}{|c}{ Clinical characteristic } & $\begin{array}{c}\text { Abnormal } \\
(n=79)\end{array}$ & $\begin{array}{c}\text { Normal } \\
(n=21)\end{array}$ & $P$ \\
\hline Sex & & & 0.745 \\
\hline Female/male & $37 / 42$ & $9 / 12$ & \\
\hline Age (y) & $10.3 \pm 2.0$ & $10.8 \pm 2.0$ & 0.293 \\
\hline Age of onset (y) & $6.7 \pm 2.6$ & $7.7 \pm 2.5$ & 0.149 \\
\hline Duration of epilepsy (y) & $3.6 \pm 2.4$ & $3.2 \pm 2.1$ & 0.713 \\
\hline Seizure frequency (times/y) & $6.0 \pm 13.4$ & $1.0 \pm 1.4$ & 0.034 \\
Seizure severity & & & 0.026 \\
\hline Mild & $27(19 / 8)$ & 12 & \\
\hline Moderate & $28(16 / 12)$ & 7 & \\
\hline Severe & $24(20 / 4)$ & 2 & \\
\hline Time since last seizure & & & 0.010 \\
\hline 1 d to 1 m & $38(25 / 13)$ & 3 & \\
\hline 1 m to 1 y & $14(11 / 3)$ & 6 & \\
\hline 1 y & $27(19 / 8)$ & 12 & \\
Antiepileptic drug & & & 0.127 \\
\hline Drug naive & $11(7 / 4)$ & 3 & \\
\hline Single drug & $35(25 / 10)$ & 14 & \\
\hline Combined drugs & $33(23 / 10)$ & 4 & \\
\hline
\end{tabular}

${ }^{*}$ Data in parentheses refer to number of patients with hypo- vs. hypermetabolism. 


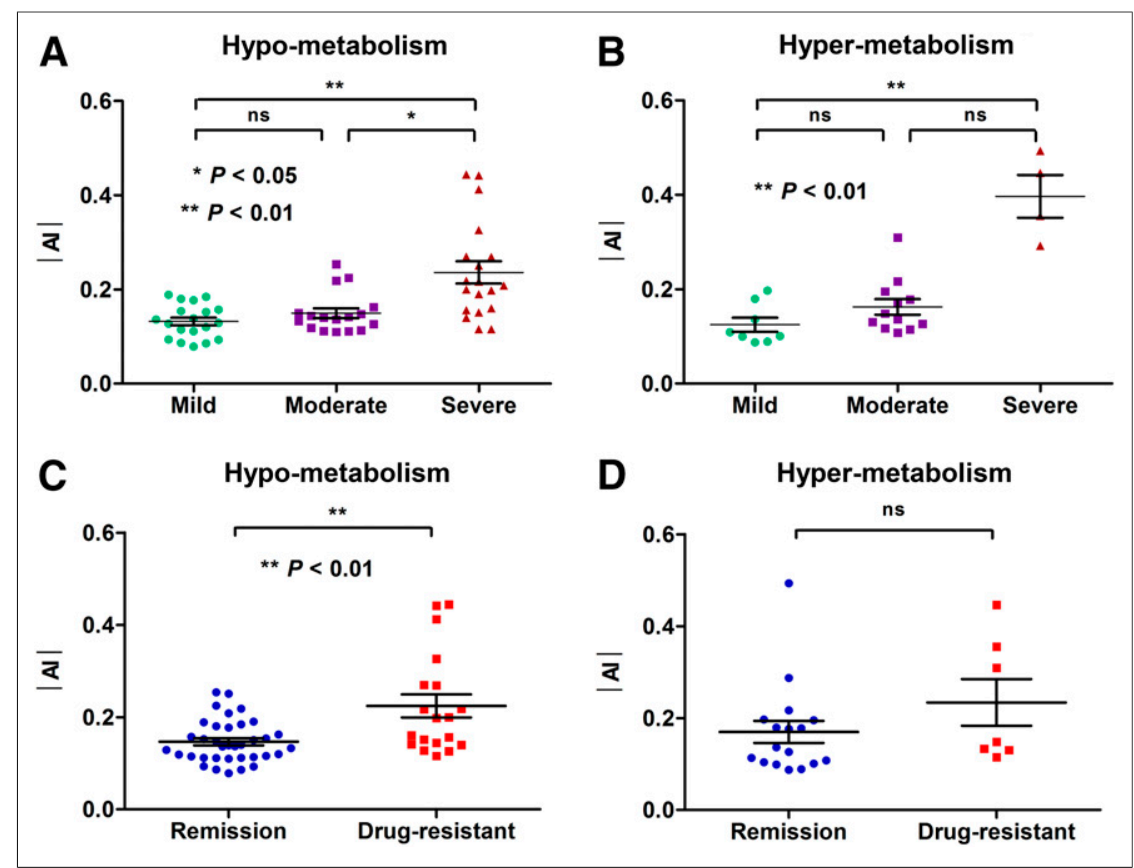

FIGURE 2. Clinical value of ${ }^{18} \mathrm{~F}-\mathrm{FDG}$ PET in severity and outcome of pediatric epilepsy. (A) In patients with hypometabolism $(n=55)$, severe group had higher value of $|A| \mid$ than mild and moderate groups $(P=0.001$ and 0.030 , respectively). (B) In patients with hypermetabolism $(n=24)$, severe group had higher value of $|\mathrm{Al}|$ than mild group $(P=0.002)$ and slightly higher value than moderate group $(P=0.051)$. (C) On the basis of follow-up, in patients with hypometabolism, drug-resistant patients had higher values of $|\mathrm{Al}|$ than seizure-free patients $(P=0.002)$. (D) In patients with hypermetabolism, drug-resistant patients had slightly higher values of $|\mathrm{Al}|$ than seizure-free patients $(P=0.209)$.

number of AEDs $(P=0.745,0.293,0.149,0.713$, and 0.127 , respectively) between patients with normal and abnormal glucose metabolism. Metabolic abnormalities were detected in 25 $(71.4 \%)$ of 35 patients with idiopathic epilepsy, in $35(77.8 \%)$ of 45 patients with cryptogenic epilepsy, and in $19(95.0 \%)$ of 20 patients with symptomatic epilepsy. No significant difference of abnormal metabolism rate was found among these 3 types of epilepsy (Fisher exact test, $P=0.109$ ).

Among patients with hypometabolism, the severe group had greater $|\mathrm{AI}|$ than the mild and moderate groups $(P<0.01$ and $<0.05$, respectively; Fig. $2 \mathrm{~A})$. In patients with hypermetabolism, the severe group had greater $|\mathrm{AI}|$ than the mild group $(P<0.01$; Fig. 2B) and slightly greater than the moderate group $(P=0.051)$. As shown in Table 4, the $|\mathrm{AI}|$ was significantly positively correlated with seizure frequency $\left(\mathrm{r}_{\mathrm{s}}=0.516, P<0.001\right.$ and $\mathrm{r}_{\mathrm{s}}=0.653, P=$ 0.001 , respectively) but negatively correlated with the time since

TABLE 4

Correlation Between $|\mathrm{Al}|$ and Clinical Severity in Pediatric Epilepsy Patients with Abnormal Glucose Metabolism

$$
(n=79)
$$

\begin{tabular}{cccccccc}
\hline & \multicolumn{2}{c}{$\begin{array}{c}\text { Seizure } \\
\text { frequency }\end{array}$} & & \multicolumn{2}{c}{$\begin{array}{c}\text { Time since } \\
\text { last seizure }\end{array}$} \\
\cline { 2 - 3 } \cline { 6 - 7 } Abnormal metabolism & $r_{\mathrm{s}}$ & $P$ & & $\mathrm{r}_{\mathrm{s}}$ & $P$ \\
\hline Hypometabolism $(n=55)$ & 0.516 & $<0.001$ & -0.477 & $<0.001$ \\
\hline Hypermetabolism $(n=24)$ & 0.653 & 0.001 & -0.621 & 0.001 \\
\hline
\end{tabular}

last seizure in patients with hypometabolism $\left(\mathrm{r}_{\mathrm{s}}=-0.477, P<0.001\right)$ and hypermetabolism $\left(\mathrm{r}_{\mathrm{s}}=-0.621, P=0.001\right)$. For patients with either hypometabolism $(P<$ $0.01)$ or hypermetabolism $(P<0.05)$, a significantly lower value of $|\mathrm{AI}|$ was found in patients with more than 1 year of time since last seizure than in those with a much shorter period since the last seizure (Supplemental Fig. 1; supplemental materials are available at http://jnm.snmjournals.org).

Stepwise multivariate linear regression analysis was performed to determine the independent contribution of individual variables (age, age of onset, duration of epilepsy, seizure frequency, time since last seizure, and number of AEDs) to the $|\mathrm{AI}|$. The only significant contributing variable was the time since last seizure, in patients both with hypometabolism $(\beta=-0.03$; $\mathrm{SEM}=0.01$; $P=0.001)$ and with hypermetabolism $(\beta=-0.05 ; \mathrm{SEM}=0.01 ; P=0.005)$.

\section{Metabolic Features of Temporal Lobe Epilepsy (TLE) and Rolandic Epilepsy}

On the basis of the clinical manifestations, EEG, MRI, and PET findings, 28 of 100 patients were found with TLE, 29 with rolandic epilepsy, 10 with frontal lobe epilepsy, and 4 with occipital lobe epilepsy.

Of the 28 patients with TLE, 26 patients $(92.9 \%)$ showed hypometabolism, 1 patient $(3.6 \%)$ had hypermetabolism in the unilateral temporal lobe, and 1 patient $(3.6 \%)$ showed an absence of any abnormal cerebral metabolism. Among the 26 patients with hypometabolism, the $|\mathrm{AI}|$ was significantly positively correlated with seizure frequency $\left(\mathrm{r}_{\mathrm{s}}=\right.$ $0.707, P<0.001)$ and negatively correlated with the time since last seizure $\left(\mathrm{r}_{\mathrm{s}}=-0.481, P<0.05\right)$.

Among the 29 patients with rolandic epilepsy, 4 metabolic patterns were found: hypermetabolism only $(n=6)$, hypermetabolism associated with hypometabolism $(n=12)$, hypometabolism only $(n=5)$, and absence of metabolic abnormality $(n=6)$. Representative images are demonstrated in Figure 3. Of 18 patients with hypermetabolism, the intensity was significantly positively correlated with seizure frequency $\left(\mathrm{r}_{\mathrm{s}}=0.597, P<0.01\right)$ but negatively correlated with the time since last seizure $\left(\mathrm{r}_{\mathrm{s}}=\right.$ $-0.569, P<0.05)$. Twenty RE patients were reported as nonlesional on MRI studies. Among the other 9 patients with abnormal MRI results, 4 patients were found with hippocampal abnormality, 2 patients with arachnoid cysts, 1 patient with an enlarged temporal horn, 1 patient with demyelination, and 1 patient with cortical dysplasia.

\section{Coregistration of PET and MR Images}

As shown in Table 1, in 39 children MRI results were abnormal, including hippocampal atrophy $(n=10)$, hippocampal sclerosis $(n=5)$, T2-weighted image signal abnormalities of hippocampus $(n=4)$, cortical dysplasia $(n=7)$, arachnoid cyst $(n=5)$, demyelination $(n=3)$, heterotopias $(n=1)$, focal cortical atrophy $(n=1)$, polymicrogyria $(n=1)$, and enlarged temporal horn $(n=2)$. Among the other 61 patients with normal MRI findings, however, 


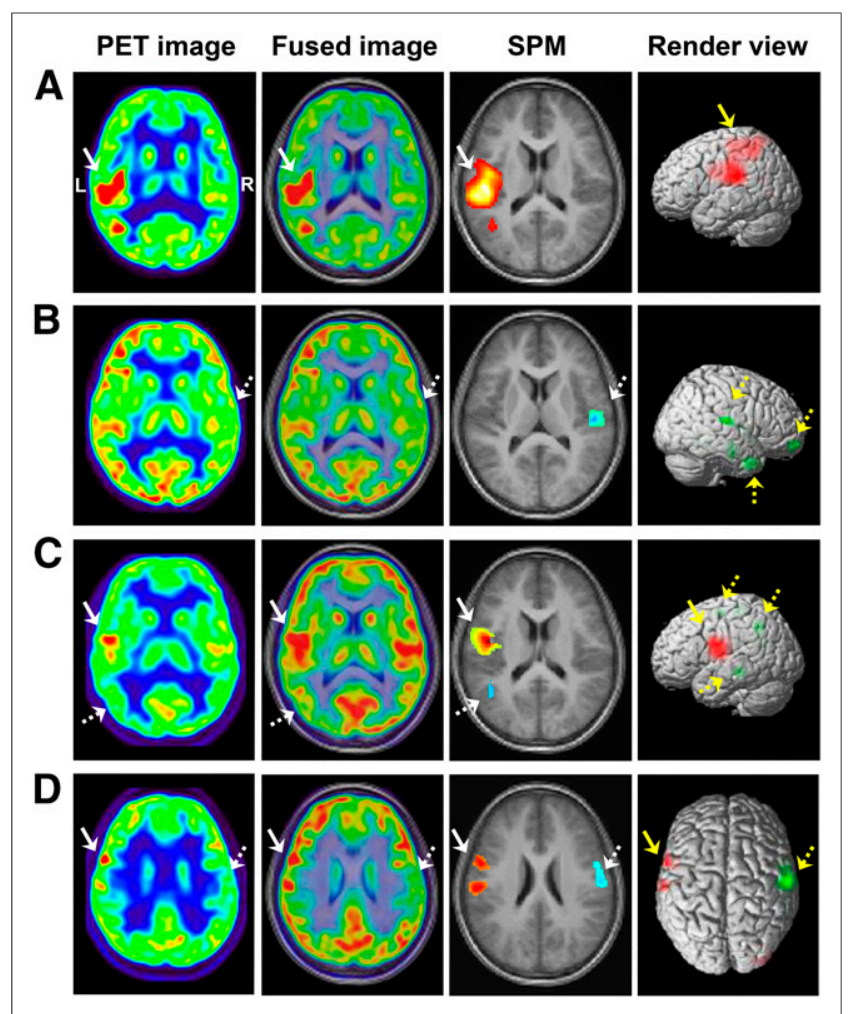

FIGURE 3. Metabolic features of rolandic epilepsy. (A) Hypermetabolism was found in left rolandic area (solid arrow). (B) Hypometabolic regions were found in right frontal and temporal lobes and rolandic areas (dashed arrow). (C) Hypermetabolism (solid arrow) with associated surrounding hypometabolism (dashed arrow) was observed in left rolandic area. (D) Hypermetabolism and its associated remote hypometabolism were found in left (solid arrow) and right (dashed arrow) rolandic areas, respectively.

after the coregistration of PET and MR images the guided second reading changed the MRI report to "subtle lesion" in 7 patients. The representative images of a 14-y-old patient are shown in Figure 4.

\section{Visual Assessment Combined with SPM Analysis and Clinical Management}

When visual assessment combined with SPM analysis was used, 79 patients $(79 \%)$ were found with abnormal metabolism, in-

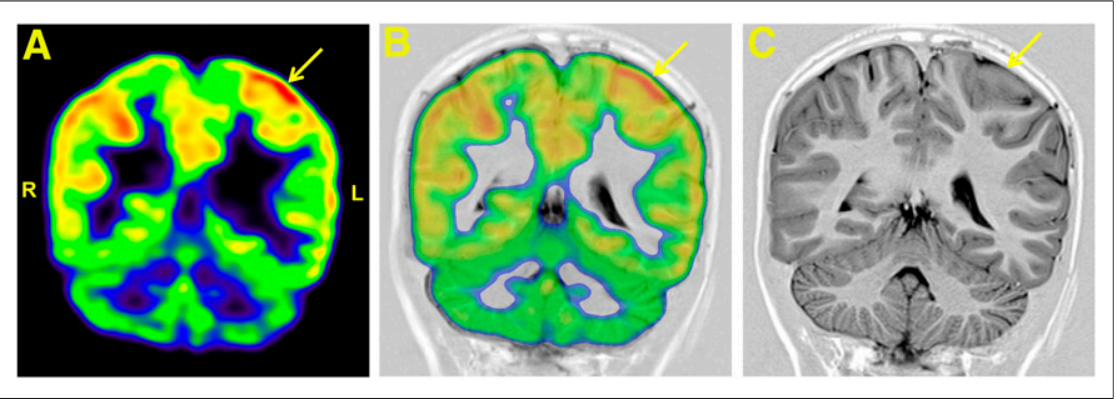

FIGURE 4. Interictal ${ }^{18} \mathrm{~F}$-FDG PET and MRI studies of 14-y-old girl who experienced 6 times of right hemifacial contraction with drooling during a 4-mo time. ${ }^{18} \mathrm{~F}-\mathrm{FDG}$ PET image (A) and PET/ MR image (B) coregistration guided second reading of MR images, in which previously missed cortical thickening in the left parietal lobe (solid arrow) on T1-weighted image (C) was detected. cluding 55 with hypometabolism and 24 with hypermetabolism (Table 4).

In patients with either hypometabolism $(P<0.01$; Fig. $2 \mathrm{C})$ or hypermetabolism ( $P=0.209$; Fig. 2D), higher values of $|\mathrm{AI}|$ were found in drug-resistant patients than in those with seizure remission. Higher values of $|\mathrm{AI}|$ were found in patients with add-on therapy than in those with unchanged therapy or withdrawal (both $P<0.01$; Fig. 5A), and slightly higher values of $|\mathrm{AI}|$ were observed in patients who showed persistence/progression than in those who showed improvement $(P=0.051$; Fig. 5B).

There were 11 patients who underwent the post-1-y follow-up PET studies. A significant change of $|\mathrm{AI}|(\%)$ was found in patients with improvement compared with those with persistence or progression $(P<0.01$; Fig. 5C).

\section{DISCUSSION}

We retrospectively evaluated the results of ${ }^{18} \mathrm{~F}-\mathrm{FDG}$ PET, MRI, and EEG in 100 nonsurgical epilepsy patients. We found that the visual assessment combined with SPM analysis could be an effective approach for identifying abnormal cerebral metabolic changes. Increased or decreased values of $|\mathrm{AI}|$ on ${ }^{18} \mathrm{~F}$-FDG PET images could be applied as an imaging biomarker for clinical evaluation of nonsurgical epilepsy. Patients with a prolonged period of seizure freedom may have more subtle (or no) metabolic abnormalities on PET. The shorter interval since the last seizure, the more patients with metabolic abnormality could be detected by ${ }^{18}$ F-FDG PET imaging. To the best of our knowledge, this is the first visual assessment combined with SPM analysis of ${ }^{18}$ F-FDG PET study to evaluate the severity and clinical management in nonsurgical pediatric epilepsy patients.

The key to successful epilepsy control is defining epileptogenic foci, whether it is an apparent anatomic focus or a more functional one, such as nonlesional epilepsy. However, the visual assessment of ${ }^{18} \mathrm{~F}$-FDG PET images in the clinical application is subjective and relies on the experience and knowledge of the readers (23). Therefore, it is inevitable that the interpretation of a single image by visual assessment is variable among different observers, and subtle metabolic abnormalities of epilepsy might not be identified easily. In our study, visual assessment combined with SPM analysis of ${ }^{18}$ F-FDG PET images identified more patients with abnormalities than visual assessment or SPM alone. Even for the nonlesional report on MRI, after coregistration of ${ }^{18}$ F-FDG PET and MR images, the guided second reading could detect subtle pathologic abnormalities in 7 patients. Similarly, another group has reported that in children with MRI-negative epilepsy, coregistered ${ }^{18}$ F-FDG PET and MR images with SPM could detect subtle MRI abnormalities that were not previously recognized in 9 out 31 patients (24). Taken their and our results together, visual assessment combined with SPM analysis of coregistered ${ }^{18}$ F-FDG PET and MR images could be an objective and effective method to identify subtle metabolic abnormalities corresponding to seizure foci.

Interictal hypometabolism was one of the first clinical findings during the development of PET, and in the past years, it has been noted to have predictive value independent of EEG and MRI (25). However, 

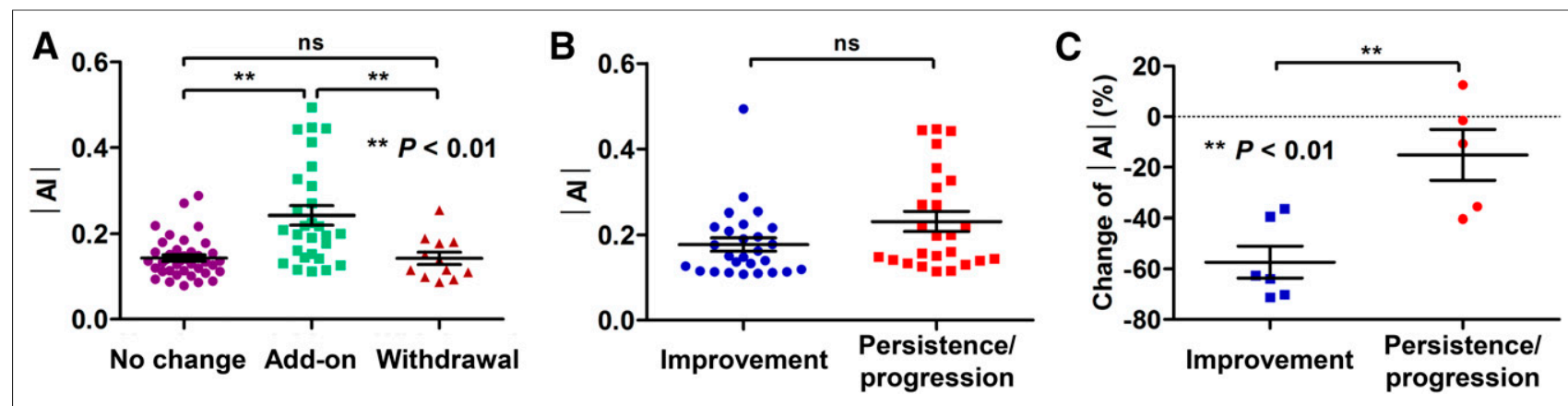

FIGURE 5. Diagnostic value of ${ }^{18} \mathrm{~F}-\mathrm{FDG}$ PET imaging during clinical course of pediatric epilepsy. (A) Patients with add-on therapy after ${ }^{18} \mathrm{~F}-\mathrm{FDG}$ PET study had higher values of $|\mathrm{Al}|$ than those with unchanged therapy or withdrawal $(P=0.006$ and $<0.001$, respectively), but no significant difference in $|\mathrm{Al}|$ was found between patients with unchanged therapy and those at withdrawal $(P=1.0)$. (B) Improvement course was defined as remaining seizure-free throughout follow-up of patients in moderate or severe groups; and persistence or progression course was defined as being drug-resistant throughout follow-up. Patients with persistence or progression course had slightly higher values of $|\mathrm{Al}|$ than those with improvement $(P=0.051)$. (C) Of 11 patients who also underwent post-1-y follow-up PET scans, significant change of $|A| \mid(\%)$ was found in patients with clinical improvement compared than with persistence or progression $(P=0.005)$. n.s. $=$ not significant.

the exact mechanism underlying hypometabolism is not entirely understood. In our nonsurgical pediatric population, we found that $92.9 \%$ of TLE patients showed hypometabolism, $3.6 \%$ had hypermetabolism in the unilateral temporal lobe, and 3.6\% showed an absence of any abnormal cerebral metabolism. A previous study has demonstrated that, despite the normal appearance of the MRI of the brain, about $40 \%$ of patients exhibit hypometabolism as measured by ${ }^{18}$ F-FDG PET at the onset of cryptogenic TLE (26). The extent of cortical glucose hypometabolism can undergo dynamic changes, which is partly related to the frequency of seizures (27). Interestingly, hypermetabolism could be detected in the acute phase of epileptic syndrome, with continuous spikes and waves during slow sleep, but disappeared in the recovery phase (28). Transient hypermetabolism was observed in 9 of 60 patients with SturgeWeber syndrome, which was considered to reflect a period of epileptogenesis (29). In an autoradiography study, ${ }^{14} \mathrm{C}-\mathrm{FDG}$ uptake was related to the general intensity of synaptic activity rather than the nature of excitation or inhibition (30). Therefore, either strong excitations or strong inhibitions had the potential to increase cerebral metabolism.

So far, the correlation between the regional asymmetry of ${ }^{18}$ F-FDG distribution and clinical severity has not been studied in patients with hypermetabolism. In the previous studies, the value of $|\mathrm{AI}|$ higher than 0.1 or 0.15 in at least one cortical region other than the cerebellum was considered abnormal $(22,27,31)$. In our study, most patients in the mild and moderate groups had $|\mathrm{AI}|$ values lower than 0.2 . The severe group had higher values of $|\mathrm{AI}|$ than the mild and moderate groups, and the $|\mathrm{AI}|$ was positively correlated with seizure frequency in patients with hyper- or hypometabolism. Although hypermetabolism is frequently observed during the ictal state of epilepsy $(32,33)$, it can also be observed in the absence of ictal events during tracer uptake and correlates well with regions of high spike count, suggesting a state of increased epileptogenicity $(33,34)$. In view of the abundant sample size of patients with interictal hypermetabolism, we assume that the $|\mathrm{AI}|$ value on ${ }^{18} \mathrm{~F}$-FDG PET images in patients with hypermetabolism could be affected by the clinical severity and seizure frequency. Previous studies demonstrated that increased seizure frequency and severity were related to greater hypometabolism on ${ }^{18} \mathrm{~F}$-FDG PET images, in both adult and pediatric patients $(22,26)$. Therefore, hypometabolism could be reversible as the seizures come under control $(27,35)$ but worsened by continuing epileptic activity (26). In addition, because the patients showing drug resistance had significant metabolic abnormalities (especially hypometabolism) compared with those showing remission, ${ }^{18}$ F-FDG PET might provide valuable follow-up information for pediatric patients with epilepsy.

Most importantly, on the basis of the results of multivariate regression analysis, we found that the timing of the last clinical seizure (rather than seizure frequency) might primarily drive the severity of metabolic asymmetries. This finding indicated that the localizing value of ${ }^{18}$ F-FDG PET may diminish in patients with prolonged seizure freedom, and on the other hand, the clinical value of PET might be enhanced by timing the scan closer to clinical seizures. Most of our patients who underwent ${ }^{18} \mathrm{~F}-\mathrm{FDG}$ PET study within less than a month since last seizure were found to have abnormal glucose metabolism. Therefore, we recommend that the optimal timing of interictal ${ }^{18}$ F-FDG PET scanning might be from $1 \mathrm{~d}$ to $1 \mathrm{mo}$ after the last seizure.

There were several limitations to the current study. First, because this is a retrospective study, the selection bias due to specific referral patterns cannot be avoided entirely. Second, no pathologic confirmation or invasive EEG recordings were available as the gold standard to study the correlation between abnormal metabolism and epileptogenic pathology. This is inherent to our selection criteria, because nonsurgical patients are unwilling to undergo invasive procedures for obvious medical and ethical reasons. Third, EEG recording was performed $1 \mathrm{~d}$ before ${ }^{18}$ F-FDG PET scanning; however, because of the retrospective feature of this study, simultaneous EEG was not performed. Fourth, we used coregistered PET/MR images, which requires repositioning of the patient and might fail to eliminate significant changes in physiologic and pathologic condition during the 2 data acquisitions. Currently, ${ }^{18}$ F-FDG PET and MR image coregistration has been recommended in the routine presurgical evaluation of epilepsy, especially in patients without lesions on MRI (36) Ideally, simultaneous imaging of PET and MR might yield benefits with regard to patient management and time saving (37). Despite these limitations, the population in this retrospective study was large enough to investigate the correlation between cerebral metabolism and severity of pediatric epilepsy, and might pave the way for a further prospective study. 


\section{CONCLUSION}

Coregistration of ${ }^{18}$ F-FDG PET/MR images with visual assessment combined with SPM analysis could provide cerebral glucose metabolic profiles in nonsurgical epilepsy patients. $|\mathrm{AI}|$ might be used for the evaluation of clinical severity and progress in these patients. Patients with a prolonged period of seizure freedom may have more subtle (or no) metabolic abnormalities on PET. The clinical value of PET might be enhanced by timing the scan closer to clinical seizures.

\section{DISCLOSURE}

This work is partly sponsored by grants from the National Key Research and Development Program of China (2016YFA0100900), National Key Basic Research Program of China (2013CB329506), National Science Foundation of China (NSFC) (no. 81425015, 81271601), and Zhejiang Provincial Natural Science Foundation of China (LR13H180001). No other potential conflict of interest relevant to this article was reported.

\section{REFERENCES}

1. Moshé SL, Perucca E, Ryvlin P, Tomson T. Epilepsy: new advances. Lancet. 2015;385:884-898.

2. Rosati A, De Masi S, Guerrini R. Antiepileptic drug treatment in children with epilepsy. CNS Drugs. 2015;29:847-863.

3. Das JC. Electroencephalogram (EEG) in the management of epilepsy in children. Mymensingh Med J. 2014;23:406-411.

4. Jones AL, Cascino GD. Evidence on use of neuroimaging for surgical treatment of temporal lobe epilepsy: a systematic review. JAMA Neurol. 2016;73:464-470.

5. Hong KS, Lee SK, Kim JY, Lee DS, Chung CK. Pre-surgical evaluation and surgical outcome of 41 patients with non-lesional neocortical epilepsy. Seizure. 2002;11:184-192.

6. Carne RP, O'Brien TJ, Kilpatrick CJ, et al. MRI-negative PET-positive temporal lobe epilepsy: a distinct surgically remediable syndrome. Brain. 2004;127:22762285.

7. Boling WW, Lancaster M, Kraszpulski M, Palade A, Marano G, Puce A. Fluorodeoxyglucose-positron emission tomographic imaging for the diagnosis of mesial temporal lobe epilepsy. Neurosurgery. 2008;63:1130-1138, discussion 1138.

8. Burneo JG, Poon R, Kellett S, Snead OC. The utility of positron emission tomography in epilepsy. Can J Neurol Sci. 2015;42:360-371.

9. Perissinotti A, Setoain X, Aparicio J, et al. Clinical role of subtraction ictal SPECT coregistered to MR imaging and ${ }^{18}$ F-FDG PET in pediatric epilepsy. $J$ Nucl Med. 2014;55:1099-1105.

10. Uijl SG, Leijten FS, Arends JB, Parra J, van Huffelen AC, Moons KG. The added value of $\left[{ }^{18} \mathrm{~F}\right]$-fluoro-D-deoxyglucose positron emission tomography in screening for temporal lobe epilepsy surgery. Epilepsia. 2007;48:2121-2129.

11. Drzezga A, Arnold S, Minoshima S, et al. ${ }^{18}$ F-FDG PET studies in patients with extratemporal and temporal epilepsy: evaluation of an observer-independent analysis. J Nucl Med. 1999;40:737-746.

12. Kim YK, Lee DS, Lee SK, Chung CK, Chung JK, Lee MC. ${ }^{18}$ F-FDG PET in localization of frontal lobe epilepsy: comparison of visual and SPM analysis. $J$ Nucl Med. 2002;43:1167-1174.

13. Won HJ, Chang KH, Cheon JE, et al. Comparison of MR imaging with PET and ictal SPECT in 118 patients with intractable epilepsy. AJNR. 1999;20:593-599.

14. Fisher RS, Acevedo C, Arzimanoglou A, et al. ILAE official report: a practical clinical definition of epilepsy. Epilepsia. 2014;55:475-482.
15. Leiderman DB, Albert P, Balish M, Bromfield E, Theodore WH. The dynamics of metabolic change following seizures as measured by positron emission tomography with fludeoxyglucose F 18. Arch Neurol. 1994;51:932-936.

16. Cramer JA, Westbrook LE, Devinsky O, Perrine K, Glassman MB, Camfield C. Development of the quality of life in epilepsy inventory for adolescents: the QOLIE-AD-48. Epilepsia. 1999;40:1114-1121.

17. Kwan P, Arzimanoglou A, Berg AT, et al. Definition of drug resistant epilepsy: consensus proposal by the ad hoc Task Force of the ILAE Commission on Therapeutic Strategies. Epilepsia. 2010;51:1069-1077.

18. Dragoumi P, Tzetzi O, Vargiami E, et al. Clinical course and seizure outcome of idiopathic childhood epilepsy: determinants of early and long-term prognosis. BMC Neurol. 2013;13:206.

19. Gelfand MJ, Parisi MT, Treves ST, Pediatric Nuclear Medicine Dose Reduction Workgroup. Pediatric radiopharmaceutical administered doses: 2010 North American consensus guidelines. J Nucl Med. 2011;52:318-322.

20. London K, Howman-Giles R. Voxel-based analysis of normal cerebral $\left[{ }^{18} \mathrm{~F}\right] \mathrm{FDG}$ uptake during childhood using statistical parametric mapping. Neuroimage. 2015;106:264-271.

21. Kumar A, Juhasz C, Asano E, Sood S, Muzik O, Chugani HT. Objective detection of epileptic foci by ${ }^{18} \mathrm{~F}$-FDG PET in children undergoing epilepsy surgery. J Nucl Med. 2010;51:1901-1907.

22. Gaillard WD, Weinstein S, Conry J, et al. Prognosis of children with partial epilepsy: MRI and serial ${ }^{18}$ FDG-PET. Neurology. 2007;68:655-659.

23. Archambaud F, Bouilleret V, Hertz-Pannier L, et al. Optimizing statistical parametric mapping analysis of ${ }^{18}$ F-FDG PET in children. EJNMMI Res. 2013;3:2.

24. Rubí S, Setoain X, Donaire A, et al. Validation of FDG-PET/MRI coregistration in nonlesional refractory childhood epilepsy. Epilepsia. 2011;52:2216-2224.

25. Struck AF, Hall LT, Floberg JM, Perlman SB, Dulli DA. Surgical decision making in temporal lobe epilepsy: a comparison of $\left[{ }^{18} \mathrm{~F}\right]$ FDG-PET, MRI, and EEG. Epilepsy Behav. 2011;22:293-297.

26. Matheja P, Kuwert T, Ludemann P, et al. Temporal hypometabolism at the onset of cryptogenic temporal lobe epilepsy. Eur J Nucl Med. 2001;28:625-632.

27. Benedek K, Juhasz C, Chugani DC, Muzik O, Chugani HT. Longitudinal changes in cortical glucose hypometabolism in children with intractable epilepsy. J Child Neurol. 2006;21:26-31.

28. De Tiège X, Ligot N, Goldman S, Poznanski N, de Saint Martin A, Van Bogaert P. Metabolic evidence for remote inhibition in epilepsies with continuous spike-waves during sleep. Neuroimage. 2008;40:802-810.

29. Alkonyi B, Chugani HT, Juhasz C. Transient focal cortical increase of interictal glucose metabolism in Sturge-Weber syndrome: implications for epileptogenesis. Epilepsia. 2011;52:1265-1272.

30. Bruehl C, Witte OW. Cellular activity underlying altered brain metabolism during focal epileptic activity. Ann Neurol. 1995;38:414-420.

31. Gaillard WD, Kopylev L, Weinstein S, et al. Low incidence of abnormal ${ }^{18}$ FDGPET in children with new-onset partial epilepsy: a prospective study. Neurology. 2002;58:717-722.

32. Meltzer CC, Adelson PD, Brenner RP, et al. Planned ictal FDG PET imaging for localization of extratemporal epileptic foci. Epilepsia. 2000;41:193-200.

33. Hur YJ, Lee JS, Lee JD, Yun MJ, Kim HD. Quantitative analysis of simultaneous EEG features during PET studies for childhood partial epilepsy. Yonsei Med J. 2013;54:572-577.

34. Bansal L, Miller I, Hyslop A, Bhatia S, Duchowny M, Jayakar P. PET hypermetabolism in medically resistant childhood epilepsy: incidence, associations, and surgical outcome. Epilepsia. 2016;57:436-444.

35. Matheja P, Weckesser M, Debus O, et al. Drug-induced changes in cerebral glucose consumption in bifrontal epilepsy. Epilepsia. 2000;41:588-593.

36. Fernández S, Donaire A, Seres E, et al. PET/MRI and PET/MRI/SISCOM coregistration in the presurgical evaluation of refractory focal epilepsy. Epilepsy Res. 2015;111:1-9.

37. Heiss WD. Hybrid PET/MR imaging in neurology: present applications and prospects for the future. J Nucl Med. 2016;57:993-995. 